Pacific Journal of Mathematics

ON 2-TRANSITIVE COLLINEATION GROUPS OF FINITE 


\title{
ON 2-TRANSITIVE COLLINEATION GROUPS OF FINITE PROJECTIVE SPACES
}

\author{
WILLIAM M. KANTOR
}

In 1961, A. Wagner proposed the problem of determining all the subgroups of $P \Gamma L(n, q)$ which are 2-transitive on the points of the projective space $P G(n-1, q)$, where $n \geqq 3$. The only known groups with this property are: those containing $P S L(n, q)$, and subgroups of $P S L(4,2)$ isomorphic to $A_{7}$. It seems unlikely that there are others. Wagner proved that this is the case when $n \leqq 5$. In unpublished work, D. G. Higman handled the cases $n=6,7$. We will inch up to $n \leqq$ 9. Our result is that nothing surprising happens. The same is true if $n=r^{\alpha}+1$ for a prime divisor $r$ of $q-1$.

One of Wagner's results is that it suffices to only consider subgroups of $P G L(n, q)$. Once this is done, it becomes simpler to view the problem as one concerning linear groups: find all those subgroups $G$ of $G L(n, q)$ which are 2-transitive on the 1-spaces of the underlying vector space $V$. Our approach is based primarily on three facts. (1) Wagner showed that the global stabilizer in $G$ of any 3-space of $V$ induces at least $S L(3, q)$ on that 3 -space. (2) Unless $G \geqq S L(n, q)$ or $n=4$, $q=2$, and $G \approx A_{7}$, no nontrivial element of $G$ can fix every 1-space of some $n$-2-space of $V$. (3) $G \leqq S L(n, q)$ if $|G|$ is divisible by a prime which is a primitive divisor of $q^{m}-1$ for a suitable $m \leqq n-2$.

Wagner's results are in [10]. Higman's result, and the case $n=$ $2^{\alpha}+1$ and $q$ odd, are mentioned by Dembowski [1], p. 39. The result mentioned above in (2) is an easy consequence of results of Wagner. The idea used in (3) is due to Perin [8] and, independently, to G. Hare and E. Shult.

I am indebted to G. Seitz for several helpful remarks.

2. Notation and preliminaries. As already mentioned, we will be dealing with linear groups. Let $V$ be an $n$-dimensional vector space over $G F(q)$. We write $G L(V)=G L(n, q)$ and $S L(V)=S L(n, q)$. It will be convenient to regard everything as taking place in the relative holomorphic $V \cdot G L(V)$. For any subgroups $K, L$ of this semidirect product we can then consider the normalizer $N_{L}(K)$ and centralizer $C_{L}(K)$. If $L \leqq G L(V)$ and $W$ is an $L$-invariant subspace of $V$, we write $L^{W}=L / C_{L}(W)$ for the subgroup of $G L(W)$ induced by $L$. $C_{L}(V / W)$ and $L^{V / W}$ are defined similarly. For any group $G$, as usual $G^{\prime}$ is its commutator subgroup, $Z(G)$ its center, and $\Phi(G)$ its Frattini subgroup. 
A group $A$ is said to be involved in a group $B$ if $A \approx C / D$ with $B \geqq C \unrhd D$.

(2.1) If $R \leqq G L(V)$ has prime power order and $(|R|, q)=1$, then $V=C_{V}(R) \oplus[V, R]$, where $[V, R]=\langle v-v r \mid v \in V, r \in R\rangle$ is $N_{G L(V)}(R)$ invariant.

Proof. [3], p. 177.

(2.2) Let $R \leqq G L(V)$ have prime power order with $(|R|, q)=1$. Let $W$ be an $R$-invariant subspace. Then $\operatorname{dim} C_{V}(R)=\operatorname{dim} C_{W}(R)+$ $\operatorname{dim} C_{V / W}(R)$.

Proof. [3], p. 187, or (2.1).

Both (2.1) and (2.2) will be used frequently, generally without reference.

A primitive divisor of $q^{k}-1$ is a prime $r$ satisfying $r \mid q^{k}-1$ but $r \nmid q^{i}-1$ for $1 \leqq i<k$; clearly $k \mid r-1$.

(2.3) (i) If $q$ is a prime power and $\dot{k} \geqq 2$, then $q^{k}-1$ has a primitive divisor unless $k=6, q=2$, or $k=2$ and $q$ is a Mersenne prime.

(ii) Let $r$ be a primitive divisor of $q^{k}-1$, and let $R$ be an $r$ subgroup of $G L(V)$ for a $G F(q)$-space $V$. If $C_{V}(R)=0$, then $k$ divides $\operatorname{dim} V$.

Proof. (i) [12].

(ii) This is clear if $|R| \leqq r$. Let $|R|>r$, and let $R_{1} \leqq Z(R)$ have order $r$. Then $V=W \oplus\left[V, R_{1}\right]$, where $W=C_{V}\left(R_{1}\right)$ is $R$-invariant and $C_{W}(R)=0$. By induction, $k$ divides $\operatorname{dim} W$ and $\operatorname{dim}\left[V, R_{1}\right]$.

(2.4) Suppose $\operatorname{dim} V=\alpha m, r$ is a primitive divisor of $q^{m}-1$, and $R \leqq G L(V)$ is an $r$-group such that $C_{V}(R)=0$. Then:

(i) Each noncyclic composition factor of $N=N_{G L(V)}(R)$ is involved in $\operatorname{PSL}\left(\alpha, q^{m}\right)$; and

(ii) If $R$ is abelian, each noncyclic composition factor of $N / C_{. v}(R)$ is involved in the symmetric group $S_{\alpha}$.

Proof. Write $V=W_{1} \oplus \cdots \oplus W_{\beta}$, with each $W_{i}$ a sum of $R$ isomorphic irreducible $R$-spaces and no two $W_{i}$ having isomorphic irreducible $R$-subspaces. Set $R_{i}=C_{R}\left(W_{i}\right)$. Then $Z\left(R / R_{i}\right)$ is cyclic and nontrivial; let $Z_{i}$ be its subgroup of order $r$. By $(2.3 \mathrm{ii}), \operatorname{dim} W_{i}=$ $m e_{i}$ for some $e_{i}$. Consequently, $\beta \leqq \alpha$ and $e_{i} \leqq \alpha$.

$N$ permutes the $W_{i}$. Let $K$ be the kernel of this permutation representation. Then $N / K$ is involved in $S_{\beta} \leqq S_{\alpha}$, and hence in $G L\left(\alpha, q^{m}\right)$. 
Set $K_{i}=N_{G L\left(W_{i}\right)}\left(Z_{i}\right)$. Then $K$ is contained in $K_{1} \times \cdots \times K_{\beta}$. Moreover, $K_{i}$ is contained in $\Gamma L\left(e_{i}, q^{m}\right)$. This proves (i).

Now assume that $R$ is abelian. Then $R / R_{i}$ is a cyclic group normalized by $K$. Since $\cap R_{i}=1$, it follows that $K / C_{K}(R)$ is abelian. Since $N / K$ is involved in $S_{\alpha^{\prime}}$ this proves (ii).

(2.5) Let $q$ be odd, and let $H \leqq G L(V)$. Suppose that $H \unrhd A \neq 1$, where $A$ is an elementary abelian 2 -group. Set

$$
m=\min \left\{\left|H: N_{H}(B)\right||B<A,| A: B \mid=2\right\} .
$$

Then $m \leqq \operatorname{dim} V$.

Proof. (G. Seitz.) Let $\bar{V}$ be an $H$-irreducible section of $V$ on which $A$ acts nontrivially. Let $\bar{H}$ and $\bar{A}$ be the groups induced by $H$ and $A$. Then $\bar{A} \neq 1$, and the corresponding $\bar{m} \geqq m$. We may thus assume that $V=\bar{V}$ is $H$-irreducible. By Clifford's Theorem ([3], p. 70), $V=V_{1} \oplus \cdots \oplus V_{t}$ with the $V_{i}$ direct sums of $A$-isomorphic irreducible $A$-spaces, no two $V_{i}$ having a common irreducible constituent. Here $A$ induces a group of order 2 on each $V_{i}$, while $H$ is transitive on $\left\{V_{1}, \cdots, V_{t}\right\}$. Thus, $\left\{C_{A}\left(V_{i}\right) \mid i=1, \cdots, t\right\}$ is an orbit of $H$ of subgroups of $A$ of index 2 . Consequently, $t \geqq m$, so $\operatorname{dim} V \geqq m$.

(2.6) Let $L$ be a finite group and $K \triangleleft L$ with $L / K$ simple. Suppose $L$ has no proper subgroup $L_{0}$ for which $L_{0} / L_{0} \cap K \approx L / K$. Then:

(i) $K$ is nilpotent; and

(ii) Each proper normal subgroup of $L$ is contained in $K$.

Proof. (i) Let $S$ be a Sylow subgroup of $K$. By the Frattini argument, $L=K N_{L}(S)$, so our conditions on $L$ imply that $L=N_{L}(S)$.

(ii) Let $M \unlhd L$ and $M \geqq K$. Since $1 \neq M K / K \unlhd L / K, M K=L$ and hence $M=L$.

(2.7) Let $d>e \geqq 2$ and $t \geqq 1$. Then $\operatorname{PSL}(d, q)$ is not involved in $\operatorname{PSL}\left(e, q^{t}\right)$.

Proof. If $p$ is the prime dividing $q$, then $p$-Sylow subgroups of $\operatorname{PSL}(d, q)$ and $\operatorname{PSL}\left(e, q^{t}\right)$ have nilpotence class $d-1$ and $e-1$, respectively.

We now come to our main technical lemma.

(2.8) Let $q=p^{e}$, where $p$ is a prime, and $m=\operatorname{dim} V$. Suppose either $m=3,4$, or 5 , or $m=6$ and $p=2$. Let $L \leqq G L(V)$ and $H, K \triangleleft L$, where $H \leqq K, L / K \approx P S L(3, q)$, and $L / H \approx P S L(3, q)$ or $S L(3, q)$. Assume that $L$ has no proper subgroup $L_{0}$ for which $L_{0} / L_{0} \cap K \approx P S L(3, q)$. Finally, assume: (\#) If $1 \neq h \in H$ and $p \nmid|h|$, then $\operatorname{dim} C_{V}(h) \leqq m-3$. 
Then there are $L$-invariant subspaces $X, Y$ with $X>Y$ such that the following hold. or $K$.

(a) $K=P \times C$ with $P$ a $p$-group, $|C|=(3, q-1)$, and $H=P$

(b) $L / P \approx S L(3, q)$.

(c) $P^{V / X}, P^{X / Y}$ and $P^{Y}$ are all 1.

(d) $\operatorname{dim} X / Y=3$ and $L^{X / Y}=S L(X / Y)$.

(e) If $m \leqq 5$ and $q \neq 2$, then $L^{V / X}$ and $L^{Y}$ are 1. Moreover, some element $g$ of order $p$ in the center of a $p$-Sylow subgroup of $L$ satisfies $\operatorname{dim} C_{V}(g) \geqq m-2$, and even $\operatorname{dim} C_{V}(g)=m-1$ if $P=1$.

Proof. Everything is obvious if $m=3$, so assume $m>3$. We will proceed by a series of steps.

(i) Clearly $L=L^{\prime}$. We can apply (2.6) to $L$. In particular, $K$ is nilpotent.

(ii) Suppose that there are $L$-invariant subspaces $V_{1}, V_{2}$ with $V_{1} \geqq V_{2}$ and $\operatorname{dim} V_{1} / V_{2} \leqq 2$. We claim that $L$ centralizes $V_{1} / V_{2}$. For, $C_{L}\left(V_{1} / V_{2}\right) \unlhd L$, and since $L^{V_{1} / V_{2}}$ does not have $P S L(3, q)$ as a homomorphic image, (2.6) implies that $C_{L}\left(V_{1} / V_{2}\right)=L$.

(iii) Next, suppose that there are $L$-invariant subspaces $X, Y$ with $X>Y, \operatorname{dim} X / Y=3$ and $L^{X / Y} \neq 1$. We claim that (a)-(e) hold.

Arguing as in (ii) we find that $L^{X / Y}=S L(X / Y)$, while $L^{V / X}$ and $L^{Y}$ are both 1 or $S L(3, q)$. Write $K=P \times C$ with $P$ a $p$-group and $C$ a $p^{\prime}$-group. $C$ induces a group of $\operatorname{order} 1$ or $(3, q-1)$ on $V / X, X / Y$, and $Y$. By (2.2), (a) holds unless $|C|=9$ and $m=6$. However, in this case $C \leqq Z(L)$, so $L / P=(L / P)^{\prime}$ is a central extension of $S L(3, q)$ by a group of order 9 , and this is impossible [2].

Thus, (a), (b), (c), and (d) hold.

Now let $m \leqq 5$. Then $\operatorname{dim} V / X$ and $\operatorname{dim} Y$ are $\leqq 2$, so $L^{V / X}$ and $L^{Y}$ are 1 by (ii). If $P \neq 1$ then, by (c), each $g \neq 1$ in $P$ satisfies $\operatorname{dim} C_{V}(g) \geqq m-2$.

Suppose $P=1$, so $L \approx S L(3, q)$. By results of Higman [4], $\S 5$, if $q \neq 2$ then there is an $L$-invariant 3 -space $T$, and each element of $L$ inducing a transvection on $T$ is a transvection of $V$. This proves (e).

(iv) From now on we assume that $m$ and $L$ are chosen with $m$ minimal such that (2.8) is false. Then $m>3$.

$L$ is irreducible on $V$. For otherwise, there is an $L$-invariant subspace $W$ with $V>W>0$.

Then $L^{W} \neq 1$ and $L^{V / W} \neq 1$. For suppose, say, that $L^{V / W}=1$. Consider $L^{W}, K^{W}$, and $H^{w}$. By (2.2), (\#) is inherited by $L^{W}$. Also, if $L_{0} \leqq L$ and $L_{0}^{W} / L_{0}^{W} \cap K^{W} \approx P S L(3, q)$ then $L_{0} K / K \approx L_{0} / L_{0} \cap K$ has $\operatorname{PSL}(3, q)$ as a homomorphic image, so that $L_{0} K=L$ and hence $L_{0}=L$. 
Consequently, $L^{W}$ satisfies the hypotheses of (2.8). Then we can find subspaces $X$ and $Y$ of $W$ such that (iii) applies, whereas (2.8) is assumed false. Thus, $L^{W} \neq 1$ and $L^{V / W} \neq 1$.

By (ii) we must have $m=6$ and $\operatorname{dim} W=3$. Then (iii) again applies, and this is again impossible.

(v) By (iv) and the nilpotence of $K,(|K|, q)=1$.

$K$ is not central in $L$. For suppose $K \leqq Z(L)$. Since $L=L^{\prime}, L$ is a homomorphic image of the covering group of $\operatorname{PSL}(3, q)$. Then $L$ is $\operatorname{PSL}(3, q)$ or $S L(3, q)$ (see, e.g., [2]).

On the other hand, $L$ has an irreducible $G F(q)$-representation of degree $m$, where $4 \leqq m \leqq 6$ and $q$ is even if $m=6$. No such representation exists by [7] and [9].

(vi) Let $r$ be a prime and $R_{1}$ an $r$-Sylow subgroup of $K$ such that $R_{1} \not Z Z(L)$. Set $R=R_{1} \cap H$. Then $R \geqq Z(L)$ and $R \triangleleft L$.

Let $A$ be a characteristic elementary abelian subgroup of $R$. By (\#), $|A| \leqq r^{m-3}$.

We claim that $A \leqq Z(L)$. For otherwise, $L$ has a nontrivial $G F(r)$-representation of degree $\leqq m-3 \leqq 3$. By $(2.6$ ii $), P S L(3, q)$ is involved in $G L(3, r)$. Thus, $q=2$ and $r \neq 3$. Since $A$ is a noncyclic elementary abelian subgroup of $G L(6,2),|A|=7^{2}$. Then $L$ acts transitively on $A-\{1\}$. However, not all elements of $A-\{1\}$ are conjugate in $G L(6.2)$.

Thus, $A \leqq Z(L)$. In (iv), $|A|=r$. In particular, $Z(R)$ is cyclic.

(vii) Suppose $r \nmid q-1$. By (vi), $R \leqq G L(6, q)$ is nonabelian, so $r=3 \mid q+1$ and $m=6$. Moreover, $R \triangleright B$ with $|R: B|=3$ and $B$ abelian. By (vi) we can find $B_{1} \neq B$ with $R \triangleright B_{1},\left|R: B_{1}\right|=3$, and $B_{1}$ abelian. Then $B \cap B_{1} \leqq Z(R)$ and $|R / Z(R)| \leqq 9$. Consequently, $L$ centralizes $Z(R), R / Z(R)$, and hence also $R$, which is not the case.

Thus, $r \mid q-1$. In (iv), $A \leqq L \cap Z(G L(V)) \leqq Z(S L(V))$, so $r \mid(q-1, m)$.

There are now just three possibilities: $m=4, r=2 ; m=5, r=5$; and $m=6, r=3$.

(viii) Let $m=4, r=2$. By (vii), $-1 \in R$. There is an involution $t \neq-1$ in $R$. Either $\operatorname{dim} C_{V}(t) \geqq 2$ or $\operatorname{dim} C_{V}(-t) \geqq 2$. This contradicts(借).

(ix) Let $m=5, r=5$. A 5-Sylow subgroup of $G L(5, q)$ has a normal abelian subgroup of index 5 (the "diagonal subgroup"). Thus, we can find $B \leqq R$ with $B$ abelian and $|R: B|=1$ or 5 . By (vi), $|R: B|$ is 5 and $B$ is not characteristic in $R$. Let $B_{1}<R, B_{1} \neq B$, satisfy the same conditions as $B$. Then $B_{1} \cap B \leqq Z(R)$ and $|R: Z(R)| \leqq 5^{2}$. By (vi), $Z(R)$ is cyclic, so $L$ centralizes $Z(R), R / Z(R)$, and hence also $R$, which is not the case.

(x) Finally, let $m=6, r=3$, and $q=2^{i}$. Here $3 \mid q-1$. On the one hand, $L / C_{L}(R / \Phi(R))$ can be regarded as a subgroup of $G L(e, 3)$ for some $e$; on the other hand, using (2.6) and $(|K|, q)=1$, we 
find that this group has an elementary abelian 2-subgroup of order $q^{2}$ whose normalizer is transitive on the nontrivial elements. By (2.5), $e \geqq q^{2}-1$. However, a 3-Sylow subgroup of $S L(6, q)$ has order $\leqq 3(q-1)^{6}$. Thus, $3^{q^{2}-1} \leqq 3^{e} \leqq|R|<3 q^{6}$, and since $q \geqq 4$ this is ridiculous.

This contradiction completes the proof of (2.8).

3. Wagner's results and some corollaries. Let $V$ be $n$-dimensional over $G F(q), n \geqq 3$, and let $G \leqq G L(V)$ be 2-transitive on 1-spaces.

(3.1) For each 3 -space $T, N_{G}(T)^{T} \geqq S L(T)$.

Proof. Wagner [10], p. 417.

(3.2) If $n \leqq 5$ then $G \geqq S L(V)$, unless $n=4, q=2$, and $G \approx A_{7}$.

Poof. Wagner [10], p.422.

(3.3) For each $n$-1-space $W, N_{G}(W)$ is 2-transitive on the 1-spaces of $V$ not in $W$.

Proof. [6], p. 6.

(3.4) If $G$ has an element $g \neq 1$ such that $\operatorname{dim} C_{V}(g) \geqq n-2$, then $G \geqq S L(V)$ or $n=4, q=2$, and $G \approx A_{7}$.

Proof. We may assume that $|g|$ is prime and $n>5$. Since $\operatorname{dim}[V, g] \leqq 2$ and $g$ centralizes $V /[V, g]$, there is a 3 -space $T>[V, g]$ such that $g^{T} \neq 1$. Then $1 \neq C_{G}(V / T)^{T} \unlhd N_{G}(T)^{T}$. By (3.1), $C_{G}(V / T)^{T} \geqq$ $S L(T)$. Choose $g^{\prime} \in C_{G}(V / T)$ with $\left|g^{\prime}\right| \mid q+1$ and $\operatorname{dim} C_{T}\left(g^{\prime}\right)=1$. Then $\operatorname{dim} C_{V}\left(g^{\prime}\right)=n-2$.

We may thus assume that $(|g|, q)=1$. Since $g^{[V, g]} \neq 1$, as before $C_{G}(V / T)^{T} \geqq S L(T)$ for each 3 -space $T>[V, g]$. By the 2-transitivity of $G$, this holds for every 3 -space of $V$.

Choose $m \leqq n$ maximal with repect to $C_{G}(V / U)^{U} \geqq S L(U)$ for all $m$-spaces $U$. Suppose $m<n$. By Wagner [10], p. 420, $m \leqq n-2$. Take any subspace $W$ of dimension $m+1$ or $m+2$. For each $m$-space $U<W, C_{G}(V / U)$ fixes $W$ and centralizes $V / W$, while $C_{G}(V / U)^{U} \geqq$ $S L(U)$. By Wagner [10], p. 420, and (3.2), $C_{G}(V / W)^{W} \geqq S L(W)$ for each $m+1$-space $W$. This contradicts the maximality of $m$.

(3.5) Let $s$ be a prime and $S$ an $s$-group maximal with respect to $\operatorname{dim} C_{V}(S) \geqq 3$. Then $N_{G}(S)$ is 2 -transitive on the 1 -spaces of $C_{V}(S)$.

Proof. Take any 3 -space $T \leqq C_{V}(S)$. Then $S$ is Sylow in $C_{G}(T)$. By the Frattini argument and (3.1), $\left(N_{G}(S) \cap N_{G}(T)\right)^{T}=N_{G}(T)^{T} \geqq$ $S L(T)$. Our assertion follows immediately. 
4. The case $n=r^{\alpha}+1$. There is one very easy case of our problem.

(4.1) THEOREM. Let $r$ be a prime divisor of $q-1$, and let $\alpha \geqq 1$. Then every collineation group of $P G\left(r^{\alpha}, q\right)$ which is 2-transitive on points contains $\operatorname{PSL}\left(r^{\alpha}+1, q\right)$.

We first prove:

(4.2) Let $r$ be a prime divisor of $q-1$, and let $\alpha \geqq 1$. Let $V$ be an $r^{\alpha}$-dimensional vector space over $G F(q)$. If $G \leqq \Gamma L(V)$ is transitive on $V-\{0\}$, then $r \| G \cap Z(G L(V)) \mid$.

Proof. Let $r^{\beta}$ be the largest power of $r$ dividing $q^{d}-1$, where $d=r^{\alpha}$. Then $q$ is not an $r^{\beta}$ th power, so $r \| G \cap G L(V) \mid$.

Let $R$ be an $r$-Sylow subgroup of $G$. By [11], p. 6, each orbit of $R$ on $V-\{0\}$ has length divisible by $r^{\beta}$.

$R$ fixes no nontrivial proper subspace of $V$. For, if it did we would have $r^{\beta} \mid q^{m}-1$ with $1 \leqq m<d$. Set $e=(d, m)$. Then $r^{\beta} \mid q^{e}-1$. However, as $d / e$ is a power of $r,\left(q^{d}-1\right) /\left(q^{e}-1\right)$ is divisible by $r$, and this contradicts the definition of $r^{\beta}$.

Let $x \in Z(R) \cap G L(V)$ have order $r$. Since $r \mid q-1, x$ can be diagonalized. By the preceding paragraph, $x$ is a scalar transformation, that is, $x \in Z(G L(V))$.

(4.3) Let $r$ be a prime divisor of $q-1$, and let $\alpha \geqq 1$. Then a collineation group of the affine space $A G\left(r^{\alpha}, q\right)$ which is 2-transitive on points contains the translation group.

Proof. (4.2).

Now (4.1) follows immediately from (3.3) and (4.3).

5. Primes dividing $|G|$. We will consider the following situation in the remainder of this paper.

Let $V$ be an $n$-dimensional $G F(q)$-space, $n \geqq 6$, and $G$ be a subgroup of $G L(V)$, 2-transitive on 1-spaces, such that $G \geqq S L(V)$. We may clearly assume that $G>Z=Z(G L(V))$.

In this section let $s$ be a prime dividing $\left(|G|, q^{m}-1\right), 1<m \leqq$ $n-2$, such that $s$ is a primitive divisor of $q^{m}-1$. (5.1) is essentially due to Perin [8] and, independently, to E. Shult and G. Hare.

(5.1) If $m=n-2$ then $q=2$ and $n$ is even.

(5.2) Suppose that $n=\alpha m+\beta, \alpha<\beta \leqq m+2$, and an element of order $s$ centralizes some 3 -space $X$. Then, for some $n^{\prime}$ satisfying $5<n^{\prime}<n$ and $n^{\prime} \equiv n(\bmod m)$, there is a subgroup of $G L\left(n^{\prime}, q\right)$, not containing $S L\left(n^{\prime}, q\right)$, which is 2-transitive on the points of $P G\left(n^{\prime}-1, q\right)$. 
Clearly (5.2) has an inductive fiavor. Since the proofs are similar, we will only prove the second of the above results.

Proof of (5.2). Choose $S \leqq C_{G}(X)$ as in (3.5). Set $W=C_{V}(S)$, $W^{*}=[V, S]$, and $N=N_{G}(S)$. Then $V=W \oplus W^{*}, C_{W^{*}}(S)=0$, and $N^{W}$ is 2-transitive on 1-spaces.

Set $n^{\prime}=\operatorname{dim} W$, so $n^{\prime} \geqq 3$. By (2.3 ii), since $\beta \leqq m+2$ we have $\operatorname{dim} W^{*}=\gamma m$ with $\gamma \leqq \alpha$. Then $n^{\prime}=n-\gamma m \geqq n-\alpha m=\beta>\alpha \geqq \gamma$.

We must show that $n^{\prime}>5$ and $N^{W} \geqq S L(W)$. Deny this. Then either $N^{W} \geqq S L(W)$ or $n^{\prime}=4, q=2$, and $N^{W} \approx A_{7}$. In particular, the commutator subgroup $N^{\prime W}$ contains a nontrivial element centralizing an $n^{\prime}$-2-space.

In this situation, $C_{N^{\prime}}\left(W^{*}\right)^{W} \leqq Z(G L(W))$. For otherwise, $C_{N^{\prime}}\left(W^{*}\right)^{W} \unlhd N^{\prime W}$ implies that $C_{N^{\prime}}\left(W^{*}\right)^{W}=N^{\prime W}$. Then $C_{N^{\prime}}\left(W^{*}\right)$ has a nontrivial element $g$ centralizing an $n^{\prime}$-2-space of $W$. Hence, $\operatorname{dim} C_{V}(g) \geqq n-2$, which contradicts (3.4).

It follows that $N^{\prime W *}$ has $P S L\left(n^{\prime}, q\right)$ as a homomorphic image, unless $n^{\prime}=4$ and $q=2$, in which case $A_{7}$ may be a homomorphic image.

Since $C_{W^{*}}(S)=0$, we can apply (2.4): each noncyclic composition factor of $N^{W^{*}}$ is involved in $\operatorname{PSL}\left(\gamma, q^{m}\right)$. Since $n^{\prime}>\gamma$, by (2.7) $\operatorname{PSL}\left(n^{\prime}, q\right)$ cannot be such a composition factor. Thus, $n^{\prime}=4, q=2, \gamma \leqq 3$, and $A_{7}$ is a composition factor of $N^{\prime W^{*}}$. However, $A_{7}$ is not involved in $\operatorname{PSL}\left(3,2^{m}\right)$. This is a contradiction.

REMARK. It is useful to note that the above proof holds under slightly weaker hypotheses: $s$ is a primitive divisor of $q^{m}-1, S \neq 1$ is an $s$-subgroup of $G$ with $W=C_{V}(S)$ of dimension $n^{\prime} \geqq 3,\left(n-n^{\prime}\right) / m<$ $n^{\prime}$, and $N_{G}(S)^{W}$ is 2 -transitive on 1-spaces.

We conclude this section with two miscellaneous results.

(5.3) Assume that $G$ has a cyclic subgroup $H$ of order $q^{n}-1$ containing an $r$-Sylow subgroup of $G$ for some prime $r$ dividing $q^{2}+q+1$. Then $q=2$ and $n$ is even.

Proof. Suppose $q \neq 2$ or $q=2$ and $n$ is odd. By (2.3), $H$ is transitive on $V-\{0\}$. Thus, $H$ is transitive on the 3 -spaces fixed by its subgroup $R$ of order $r$.

On the other hand, by (3.1) each 3-space is fixed by a conjugate of $R$. Thus, $G$ is transitive on 3 -spaces, and this contradicts Perin [8] or (5.1) since $n \geqq 6$.

(5.4) Assume that $G$ has a cyclic subgroup of order $q^{n-1}-1$ fixing some $n-1$-space $W$ and transitive on $W-\{0\}$. Then $N_{G}(W)$ is 2-transitive on the 1-spaces of $W, q=2$, and $n$ is even. 
Proof. We may assume that $G-Z$ has no element fixing all 1- spaces in $W$. By [6], Lemma 7.3, $N_{G}(W)$ is 2-transitive on the 1-spaces of $W$. The result now follows from (2.3) and (5.1).

6. The case $n \leqq 9$. Let $n, V, G$, and $Z$ be as in $\S 5$, so $G \nsupseteq$ $S L(V)$. Let $p$ be the prime dividing $q$.

Assume that $6 \leqq n \leqq 9$.

(6.1) $n \neq 6$.

Proof. Suppose $n=6$. If $q=2$ then $q^{5}-1$ is a prime. By (5.4), the stabilizer of a 5-space $W$ is 2-transitive on $W-\{0\} . \quad$ By (3.2) and (3.4), $G \geqq S L(V)$, which is not the case.

Thus, $q>2$. Let $r$ be a prime dividing $q-1$.

Suppose that there is 3 -space $T$ for which $N_{G}(T)-Z$ contains an element inducing a scalar transformation of order $r$ on $T$. Using $Z$, we find that $r|| C_{G}(T) \mid$. Let $R$ be an $r$-Sylow subgroup of $C_{G}(T)$. By (3.4), $T=C_{V}(R)$. By (3.5), $N_{G}(R)^{T} \geqq S L(T)$. Also, $N_{G}(R)$ normalizes the 3-space $[V, R]$. An element of order $p$ in the center of a $p$ Sylow subgroup of $N_{G}(R)$ centralizes 2-spaces of both $C_{V}(R)$ and $[V, R]$, and hence centralizes a 4-space of $V=C_{V}(R) \oplus[V, R]$. This contradicts (3.4). Thus, no element of $G-Z$ of order $r$ has an eigenspace of dimension $>2$.

Now take any 3 -space $T$, and write $T=X \oplus Y$ with $\operatorname{dim} X=2$ and $\operatorname{dim} Y=1$. Set $F=N_{G}(X) \cap N_{G}(Y)$, so $F^{X}=G L(X)$. Take $R \leqq F$ of order $r$ with $R \nsubseteq Z$ and $R^{T} \leqq Z\left(F^{T}\right)$. By the Frattini argument, $N_{F}(R)^{X}=G L(X)$. Let $E \leqq N_{F}(R)$ be minimal with respect to $E^{X}=S L(X)$.

Since $R$ is diagonalizable and each of its eigenspaces has dimension 1 or 2 , we can write $V=X \oplus W_{1} \oplus W_{2}$ with $W_{1}>Y, \operatorname{dim} W_{i}=$ 2 , and $W_{i}$ invariant under $N_{G}(R)$. If $q \neq 3, E=E^{\prime}$ centralizes $W_{1}$, so an element of $E$ of order $p$ centralizes a 4 -space, which contradicts (3.4). If $q=3, R$ cannot have more than two eigenspaces as $|R|=$ 2 , which is again a contradiction.

(6.2) $q$ is even.

Proof. Assume that $q$ is odd. There is an involution $t \in G-Z$. Since $n \geqq 6, \operatorname{dim} C_{V}(t)$ or $\operatorname{dim} C_{V}(-t)$ is $\geqq 3$. Let $S$ be a 2-group in $G$ maximal with respect to $\operatorname{dim} C_{V}(S) \geqq 3$. Set $W=C_{V}(S)$ and $W^{*}=$ $[V, S]$, so $V=W \oplus W^{*}$. Set $M=N_{G}(S)$. By (3.5), $M^{W}$ is 2-transitive on 1-spaces. Since $M>Z$ and all involutions in $M^{W}$ centralize at most a 2 -space (by the maximality of $S$ ), $\operatorname{dim} W \leqq 4$. Consequently, by (3.2), $M^{W} \geqq S L(W)$.

By (4.1) and (6.1), $n=7$ or 8 , so $\operatorname{dim} W^{*} \leqq 5$. 
We claim that $C_{M}\left(W^{*}\right)^{W} \leqq Z(G L(W))$. For otherwise, $C_{M}\left(W^{*}\right)^{W} \unlhd$ $M^{W}$ yields $C_{M}\left(W^{*}\right)^{W} \geqq S L(W)$. Then $C_{M}\left(W^{*}\right)$ contains a nontrivial transvection of $V$, which contradicts (3.4).

Thus, $C_{M I}\left(W^{*}\right)$ is cyclic and $M^{H^{* *}}$ has $\operatorname{PSL}(W)$ as a homomorphic image.

Suppose that $\operatorname{dim} W=4$. Then $\operatorname{dim} W^{*}=3$ or 4 . Use of $M^{\prime W^{*}}$ yields $\operatorname{dim} W^{*}=4$ and $M^{\prime W^{*}} \geqq S L\left(W^{*}\right)$. If $g \neq 1$ is in the center of a $p$-Sylow subgroup of $M^{\prime}$ then $g^{W}$ and $g^{W *}$ are transvections, and this contradicts (3.4).

Thus, $\operatorname{dim} W=3$. Let $L \leqq M$ be minimal with respect to having $P S L(3, q)$ as a homomorphic image. Let $H=C_{L}(W) \leqq K \triangleleft L$ with $L / K \approx P S L(3, q)$. Then (2.8) applies to $W^{*}, L^{W^{*}}, K^{W^{*}}$, and $H^{W^{*}}$.

Choose $g \in L$ so that $g^{W^{*}}$ is as in $(2.8 \mathrm{e})$. If $g \in H=C_{L}(W)$, then $\operatorname{dim} C_{V}(g) \geqq n-2$. If $H^{W^{*}}=1$ then $H=1$, and both $g^{W}$ and $g^{W^{*}}$ are transvections, so once again $\operatorname{dim} C_{V}(g) \geqq n-2$. In either case we have contradicted (3.4).

(6.3) $n \neq 7,8$.

Proof. Let $n=7$ or 8 . Fix a prime $r \mid q+1$.

Take any 3 -space $T$. By (3.1), $N_{G}(T)^{T} \geqq S L(T)$. Also, $N_{G}(T)$ acts on $V / T$. By (3.4), $C_{G}(V / T)^{T} \leqq Z\left(G L(T)\right.$ ) (since otherwise, $C_{G}(V / T)$ would have an element of order $r)$, so $C_{G}(V / T)$ is solvable. Thus, $N_{G}(T)^{V^{\prime} / T}$ has $P S L(3, q)$ as a composition factor. By (2.8), there is an $r$-group $R \neq 1$ in $N_{G}(T)$ such that $\operatorname{dim} C_{V / T}(R) \geqq 2$, and then $\operatorname{dim} C_{V}(R) \geqq 3$.

This contradicts (5.2) with $n=2 \cdot 2+3$ or $2 \cdot 2+4$.

(6.4) If $n=9$ then $q=2$ or 4 .

Proof. Suppose $n=9$ and $q>4$ is even.

(i) By (5.2) with $n=2 \cdot 3+3$, no nontrivial element of order dividing $\left(q^{2}+q+1\right) /(q+1,3)$ can centralize a 1 -space.

(ii) Let $T$ be any 3 -space. Let $L \leqq N_{G}(T)$ be minimal with respect to having $P S L(3, q)$ as a homomorphic image. By (3.4), $C_{G}(V / T)^{T} \leqq Z(G L(T))$, so (2.8) applies to $L^{V / T}$. Consequently, by (i) there is a 6-space $Y>T$ such that $L^{Y / T}=S L(Y / T)$ and $L^{V / Y}=S L(V / Y)$.

(iii) Let $s$ be a prime dividing $q+1$. By (ii), there is an element of order $s$ centralizing a 3-space.

Let $S$ be an $s$-group maximal with respect to $\operatorname{dim} C_{V}(S) \geqq 3$. By (3.5), $N_{G}(S)$ is 2-transitive on the 1-spaces of $C_{V}(S)$. In view of (i), it follows from (3.2), (6.1), and (6.3) that $\operatorname{dim} C_{V}(S)=3$.

Let $T=C_{V}(S)$ in (ii), and choose $L \leqq N_{G}(S)$ there. By (i) and the proof of $(2.4),(L S)^{[r, S]}$ acts as a subgroup of $\Gamma L\left(3, q^{2}\right)$, with $S$ inducing scalar transformations. 
(iv) Since $q>4$, by $(2.3$ i) there is a prime $r \neq 3$ dividing $q-1$. Moreover, if $q \neq 16$ we can choose $r \neq 5$.

We claim that some element of order $r$ centralizes a 4-space. For, since $r \neq 3$, in (iii) we can find $g \in L-Z$ of order $r$ such that $g^{[V, s]}$ has an eigenspace of dimension $\geqq 4$. Consequently, some element of $\langle g, Z\rangle$ of order $r$ centralizes a 4-space.

(v) Let $R$ be an $r$-group maximal with respect to $\operatorname{dim} C_{V}(R) \geqq 3$; by (iv), $R \neq 1$. Set $T=C_{V}(R)$ and $T^{*}=[V, R]$. By (3.5), $N_{G}(R)^{T}$ is 2 -transitive on 1-spaces, so $\operatorname{dim} T=3$ by (i). We can thus choose $L \leqq N_{G}(R)$ in (ii).

We claim that $L R$ centralizes $R$ and that $R$ is diagonalizable. Certainly $(L R)^{r^{*}} \leqq G L\left(T^{*}\right)$. Suppose $r>5$. Then an $r$-Sylow subgroup of $G L(6, q)$ is diagonalizable, and hence abelian. By (2.4 ii) (with $m=1, \alpha=6$ ), each composition factor of $L / C_{L}(R)$ is involved in $S_{6}$. By (2.6 ii), $L=C_{L}(R)$, so $R \leqq Z(L R)$.

Consider the case $r=5, q=16$. Suppose $L>C_{L}(R)$. Then $L$ acts nontrivially on $R / \Phi(R)$, where $|R / \Phi(R)| \leqq 5^{7}$. By (2.6 ii), $16+1$ divides $|G L(7,5)|$, which is not the case.

Thus, $L$ centralizes $R$. There is an s-group $S_{0}<L$ such that $\operatorname{dim} C_{T^{*}}\left(S_{0}\right)=2$. Since $R$ normalizes $C_{T^{*}}\left(S_{0}\right)$ and $\left[T^{*}, S_{0}\right]$, it follows that $R$ is again diagonalizable. Thus, $R \leqq Z(L R)$.

(vi) $T^{*}$ is the direct sum of $R$-invariant subspaces, each invariant under $L R$. By (ii) and (v), there are 3-spaces $X$ and $X^{\prime}$ such that $T^{*}=X \oplus X^{\prime}, R^{X}$ and $R^{X^{\prime}}$ consist of scalar transformations, $L^{X}=$ $S L\left(X^{\prime}\right)$, and $L^{X^{\prime}}=S L\left(X^{\prime}\right)$.

Consequently, for each $h \in R, \operatorname{dim} C_{V}(h)=3,6$, or 9 .

(vii) By (iv), there is an $r$-group $R_{1} \neq 1$ maximal with respect to $\operatorname{dim} C_{V}\left(R_{1}\right) \geqq 4$. By (vi), $W=C_{V}\left(R_{1}\right)$ has dimension 6 . Set $M=$ $N_{G}\left(R_{1}\right)$.

Take any 3 -space $T<W$. Let $R \geqq R_{1}$ be an $r$-Sylow subgroup of $C_{G}(T)$. If $R=R_{1}$ then $N_{M}(T)^{T} \geqq S L(T)$ by the Frattini argument. If $R>R_{1}$ then the choice of $R_{1}$ implies that $C_{V}(R)=T$, and hence that $R$ is an $r$-group maximal with respect to $\operatorname{dim} C_{V}(R) \geqq 3$; by (v), $C_{G}(R)^{T} \geqq S L(T)$, so again $N_{M}(T)^{T} \geqq S L(T)$.

Consequently, $M^{W}$ is 2 -transitive on 1 -spaces. Then $\left(q^{6}-1\right) /(q-1)$ divides $|G|$, and this contradicts (5.2).

(6.5) If $n=9$ then $q \neq 4$.

Proof. Suppose $n=9$ and $q=4$. We will try to imitate the proof of (6.4) using $r=3$. Steps (i) and (ii) of that proof still hold.

We begin by showing the existence of $x \in G$ of order 3 such that $x^{y}=x^{-1}$ for some 2-element $y$. Take $T$ and $L$ as in (ii). Then we can find $x, y \in L$ with $|x|=3, y$ a 2-element, and $x^{y}=x^{-1} a, a \in C_{L}(T)$. 
By (2.8), $C_{L}(T)=P \times C$ with $P$ a 2-group and $|C|=1$ or 3 . Then $\langle x\rangle$ is Sylow in $\langle x, y\rangle P$. By the Frattini argument, some element of $\langle y\rangle P$ inverts $\langle x\rangle$, and we may assume this is $y$.

We next claim that some element of order 3 centralizes a 4 -space. For, assume that this is false, and choose $x, y$ as above. Since $q=$ $4, x$ is diagonalizable and has at most 3 eigenspaces. However, no element of $\langle x, Z\rangle-\{1\}$ centralizes a 4 -space, so $C_{V}(x)=T$ is a 3 -space and $x$ has two other 3 -dimensional eigenspaces $T_{1}, T_{2}$. Moreover, by our assumption, $C_{G}(T)$ has a cyclic 3-Sylow subgroup. Thus, by the Frattini argument, $\quad N_{G}(\langle x\rangle)^{T} \geqq S L(T)$, so $C_{G}(x)^{T} \geqq S L(T)$. Since $|G L(T): S L(T)|=3, y^{T} \in S L(T)$, so we can find $c \in C_{G}(X)$ such that $c^{-1} y \in C_{G}(T)$. Clearly $c^{-1} y$ inverts $x$, so there is an involution $t \in$ $\left\langle c^{-1} y\right\rangle$. Here, $t$ centralizes $T$ and centralizes 2-spaces of each $T_{i}$, so $\operatorname{dim} C_{V}(t) \geqq 7$. This contradicts (3.4), and proves our claim.

Now define $R, T, T^{*}$, and $L$ as in (v). We will be able to obtain a contradiction precisely as in (vi) and (vii) if we can show that $R \leqq$ $Z(L R)$ and $R$ is diagonalizable.

By (2.6), $L \triangleright K$ with $L / K \approx P S L(3,4)$ and $K$ nilpotent. By (2.2) and (2.8), $K=P \times C$ with $|C|=3$ or 9 and $P$ a 2-group; moreover, there is an $L$-invariant 3 -space $X<T^{*}$ such that $L^{X}=S L(X), L^{T^{*} / X}=$ $S L\left(T^{*} / X\right)$, and $P$ centralizes $T, X$, and $T^{*} / X$. By (3.4), no nontrivial element of $P$ centralizes a 4 -space of $T^{*}$. Consequently, $P$ is elementary abelian of order $\leqq 4^{3}$. Thus, if $P \geqq Z(L)$ then $P S L(3,4)$ is isomorphic to a subgroup of $G L(6,2)$, which is not the case ([7], [9]). Thus, $K \leqq Z(L)$.

Now suppose that $L$ acts nontrivially on $R$, and hence on $R / \Phi(R)$. Since $R \leqq G L(6,4),|R / \Phi(R)| \leqq 3^{6} \cdot 3^{2}$. Thus, $P S L(3,4)$ or $S L(3,4)$ is isomorphic to a subgroup of $G L(8,3)$. Then $G L(8,3)$ has an elementary abelian subgroup of order $4^{2}$ whose normalizer is transitive on the nontrivial elements. By (2.5), this is impossible.

Consequently, $L \leqq C_{G}(R)$. An element of $L$ of order 5 centralizes 1 -spaces of $X$ and $T^{*} / X$. It follows that $T^{*}$ is the sum of $R$-invariant 2-spaces. Thus, $R$ is diagonalizable and $R \leqq Z(L R)$. This completes the proof of $(6.5)$.

Last, and least:

(6.6) If $n=9$ then $q \neq 2$.

Proof. Suppose $n=9$ and $q=2$. Using (5.1) and (5.2) we find that $|G|=2^{\alpha} \cdot 3^{\beta} \cdot 5 \cdot 7 \cdot 17 \cdot 73$ for some $\alpha, \beta$.

Let $S$ be a 73-Sylow subgroup of $G$. By (5.3), $\left|C_{G}(S)\right|=73$. Thus, $\left|N_{G}(S)\right|=3^{\gamma} \cdot 73$ with $\gamma \leqq 2$.

By Sylow's theorem, $2^{\alpha} \cdot 3^{\beta-\gamma} \cdot 5 \cdot 7 \cdot 17 \equiv 1(\bmod 73)$. A little arithmetic shows that this is impossible. 
In view of (3.2) and the results of this section, we can now state:

THEOREM 6.7. Let $H$ be a subgroup of $P \Gamma L(n, q)$ which is 2transitive on the points of $P G(n-1, q)$. If $3 \leqq n \leqq 9$, then $H \geqq$ $\operatorname{PSL}(n, q)$ or $n=4, q=2$, and $H \approx A_{7}$.

\section{REFERENCES}

1. P. Dembowski, Finite Geometries, Springer, Berlin-Heidelberg-New York, 1968.

2. W. Feit, The current situation in the theory of finite simple groups, Proc. Int. Cong. Math. Nice, 1970, Vol. 1, 55-93.

3. D. Gorenstein, Finite Groups, Harper and Row, New York, 1968.

4. G. Hare, Thesis, Southern Illinois University, 1970.

5. D. G. Higman, Flag-transitive collineation groups of finite projective spaces, Illinois J. Math., 6 (1962), 434-446.

6. W. M. Kantor, 2-Transitive symmetric designs, Trans. Amer. Math. Soc., 146 (1969), $1-28$.

7. C. Mark, Thesis, University of Toronto, 1939.

8. D. Perin, On collineation groups of finite projective spaces, Math. Z., 126 (1972), 135-142.

9. R. Steinberg, Representations of algebraic groups, Nagoya Math. J., 22 (1963), 3356.

10. A. Wagner, On collineation groups of finite projective spaces, I. Math. Z., 76 (1961), 411-426.

11. H. Wielandt, Finite Permutation Groups, Academic Press, New York, 1964.

12. K. Zsigmondy, Zur Theorie der Potenzreste, Monatsh. f. Math. u. Phys., 3 (1892), 265-284.

Received November 6, 1971 and in revised form October 13, 1972. This research was supported in part by NSF Grant GP28420.

UNIVERSITY OF OREGON 



\section{PACIFIC JOURNAL OF MATHEMATICS}

\section{EDITORS}

RICHARD ARENS (Managing Editor)

University of California

Los Angeles, California 90024

R. A. Beaumont

University of Washington

Seattle, Washington 98105
J. DUGUNDJI*

Department of Mathematics

University of Southern California

Los Angeles, California 90007

D. Gilbarg and J. Milgram

Stanford University

Stanford, California 94305

\section{ASSOCIATE EDITORS}
E. F. BECKENBACH
B. H. NeumanN
F. WOLF
K. YosHIDA

\section{SUPPORTING INSTITUTIONS}

UNIVERSITY OF BRITISH COLUMBIA
CALIFORNIA INSTITUTE OF TECHNOLOGY
UNIVERSITY OF CALIFORNIA
MONTANA STATE UNIVERSITY
UNIVERSITY OF NEVADA
NEW MEXICO STATE UNIVERSITY
OREGON STATE UNIVERSITY
UNIVERSITY OF OREGON
OSAKA UNIVERSITY

UNIVERSITY OF BRITISH COLUMBIA

UNIVERSITY OF CALIFORNIA

MONTANA STATE UNIVERSITY

UNIVERSITY OF NEVADA

OREGON STATE UNIVERSITY

OSAKA UNIVERSITY
UNIVERSITY OF SOUTHERN CALIFORNIA

STANFORD UNIVERSITY

UNIVERSITY OF TOKYO

UNIVERSITY OF UTAH

WASHINGTON STATE UNIVERSITY

UNIVERSITY OF WASHINGTON

\section{AMERICAN MATHEMATICAL SOCIETY} NAVAL WEAPONS CENTER

* C. R. DePrima California Institute of Technology, Pasadena, CA 91109, will replace J. Dugundji until August 1974. 


\section{Pacific Journal of Mathematics}

\section{Vol. 48, No. $1 \quad$ March, 1973}

Jan Aarts and David John Lutzer, Pseudo-completeness and the product of Baire

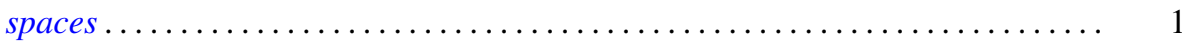

Gordon Owen Berg, Metric characterizations of Euclidean spaces ............ 11

Ajit Kaur Chilana, The space of bounded sequences with the mixed topology ..... . 29

Philip Throop Church and James Timourian, Differentiable open maps of

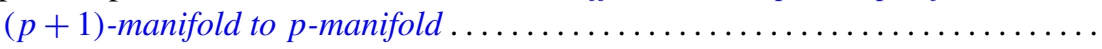

P. D. T. A. Elliott, On additive functions whose limiting distributions possess a finite

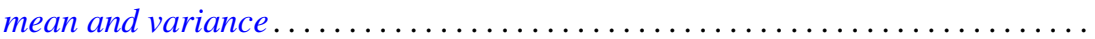

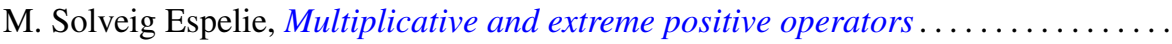

Jacques A. Ferland, Domains of negativity and application to generalized convexity

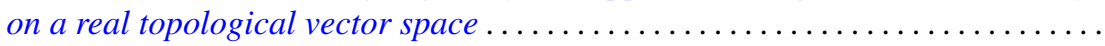

Michael Benton Freeman and Reese Harvey, A compact set that is locally holomorphically convex but not holomorphically convex ...............

Roe William Goodman, Positive-definite distributions and intertwining

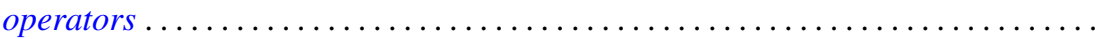

Elliot Charles Gootman, The type of some $C^{*}$ and $W^{*}$-algebras associated with

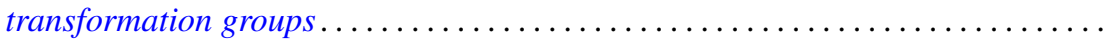

David Charles Haddad, Angular limits of locally finitely valent holomorphic

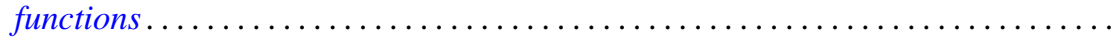

William Buhmann Johnson, On quasi-complements .

William M. Kantor, On 2-transitive collineation groups of finite projective spaces...

Joachim Lambek and Gerhard O. Michler, Completions and classical localizations of right Noetherian rings

Kenneth Lamar Lange, Borel sets of probability measures ......

David Lowell Lovelady, Product integrals for an ordinary differential equation in a Banach space

Jorge Martinez, A hom-functor for lattice-ordered groups .........

W. K. Mason, Weakly almost periodic homeomorphisms of the two sphere ....

Anthony G. Mucci, Limits for martingale-like sequences .......

Eugene Michael Norris, Relationally induced semigroups ...

Arthur E. Olson, A comparison of c-density and $k$-density ......

Donald Steven Passman, On the semisimplicity of group rings of linear groups.

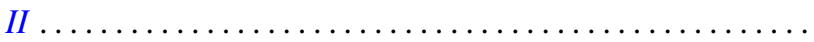

Charles Radin, Ergodicity in von Neumann algebras .

P. Rosenthal, On the singularities of the function generated by the Bergman operator of the second kind.

Arthur Argyle Sagle and J. R. Schumi, Multiplications on homogeneous spaces,

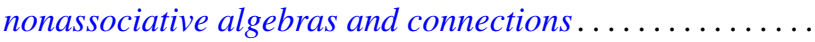

Leo Sario and Cecilia Wang, Existence of Dirichlet finite biharmonic functions on

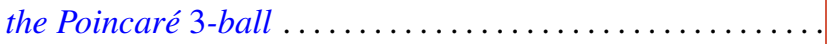

Ramachandran Subramanian, On a generalization of martingales due to Blake ..

Bui An Ton, On strongly nonlinear elliptic variational inequalities.

Seth Warner, A topological characterization of complete, discretely valued

fields. 